\title{
Linear oscillatory actuator integrated with magnetic gear for E-cutter development
}

\begin{abstract}
This paper proposes a linear oscillatory actuator integrated with a magnetic gear for E-cutter development to harvest oil palm fresh fruit bunch (FFB). The machine comprises of a linear magnetic gear which is artfully integrated with a slotless linear motor. The idea is proposed to improve the performance of existing linear actuators. The design is targeted for a optimum motor thrust, light weight, low cycle time, and reliable displacement. Most importantly, the direction of motion for both the linear motor and linear magnetic gear is opposite to each other, and this could reduce the vibration disturbance from the machine. The performance of the proposed machine is analyzed by finite element method (FEM) and the simulation results verify the machine design.
\end{abstract}

Keyword: Cutting time; Displacement; FEM analysis; Linear magnetic gear; Thrust 